\title{
Sensorless Speed Control of Five-Phase PMSM Drives with Low Current Distortion
}

\begin{abstract}
This paper introduces a design for a sensorless control of a five-phase PMSM drive working at low and zero speeds with low current distortion. The rotor position is obtained through tracking the saturation saliency by measuring the dynamic currents responses of the motor due to the IGBTs switching actions. It uses the fundamental PWM waveform obtained using the multi-phase space vector pulse width modulation only. The saliency tracking algorithm used in this paper doesn't only improve the quality of the estimated position signals but also guarantees a minimum current distortion through reducing the modifications introduced on the PWM waveform. Simulation results are provided to verify the effectiveness of the proposed strategy for saliency tracking and current distortion minimizing of a five-phase PMSM motor drive over a wide speed ranges under different load conditions.
\end{abstract}

Keywords: Sensorless, five-phase motor, multi-dimension SVPWM, THD.

\section{Introduction}

The interest in multi-phase motor drives has increased in recent years as they offer several advantages when compared to three-phase machines such as improving reliability, efficiency and torque density and reducing torque pulsations $[1,2]$. Therefore, multi-phase motor drives are extensively considered for applications related to vehicles, aerospace application (more electric craft), ship propulsion and high power applications. The modulation technique that is proposed for using in multi-phase motor drives is multi-dimension SVPWM which is based on the concept of orthogonal multi-dimensional vector space $[3,4,5,6,7,8,9$, $10,11,12]$. This modulation technique can synthesize voltage vectors both in d-q subspace and in other subspace. Many control techniques has been adopted to control the multi-phase motor drives in sensored mode such as fuzzy logic control [13], predictive current control [14].

In the last couple of years, few researches have been directed towards the sensorless control of a multiphase electrical drives. These researches focus on the model based sensorless control, direct torque control and high frequency injections $[15,16,17,18,19]$.

This paper introduces a new method to track the saturation saliency in five-phase PMSM drives to obtain the rotor position through measuring the dynamic currents responses of the motor due to the IGBTs switching actions $[20,21,22]$. It uses the fundamental PWM waveform obtained using the multi-phase space vector pulse width modulation only. The saliency tracking algorithm used in the proposed method doesn't only improve the quality of the estimated position signals but also guarantees a minimum current distortion in the motor currents through reducing the modifications introduced on the PWM waveform.

\section{Research Method}

\subsection{Five-phase converter drive topology}

Figure 1 shows the proposed five-phase converter drive topology [17]. The model of the motor is a fivephase model not a $d-q$ model to make the results more realistic. Also the saturation saliency $(2 * \mathrm{fe})$ is embedded in the motor equations.

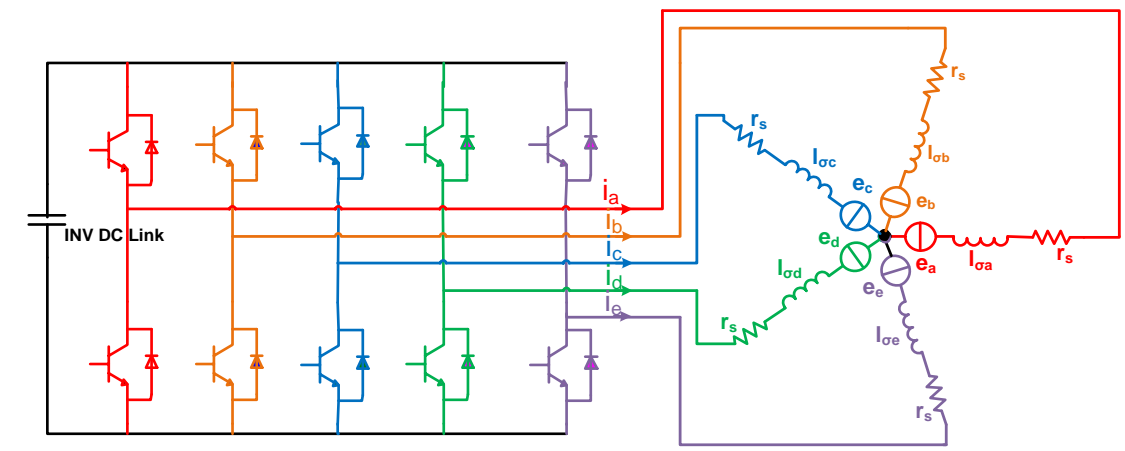

Figure 1. Five phase converter drive topology.

2.2 Multi-phase Space Vector Pulse Width Modulation

\subsubsection{Concept of Orthogonal Vector Space}


The kth order harmonics $(\mathrm{k}=5 \times \mathrm{m} \pm 2, \mathrm{~m}=1,3,5 \ldots)$ of the machine's variables such as phase voltage, phase current which do not produce any rotating MMF and are non electromechanical energy conversion related will freely flow through the windings of the five-phase motors and their amplitude will be restricted by the stator leakage impedance only [3-12, 15-19]. Hence, generation of certain low-order voltage harmonics (3rd harmonic) in the VSI output can lead to large 3rd harmonic in stator current. It is therefore important that the multi-phase VSI output is kept as close as possible to sinusoidal. To do so, the reference voltage should be mapped into two orthogonal planes. The first one is the $\mathrm{d} 1-\mathrm{q} 1$ plane that is rotating at synchronous speed and has fundamental components of the reference voltage. The second is the $\mathrm{d} 3-\mathrm{q} 3$ plane that is rotating at 3 times the synchronous speed and has the 3rd harmonic components of the reference voltage using the transformation given in (1) and (2):-

$$
\begin{aligned}
& {\left[\begin{array}{l}
d 1 \\
q 1 \\
d 3 \\
q 3
\end{array}\right]=[G]\left[\begin{array}{l}
a \\
b \\
c \\
d \\
e
\end{array}\right] . .(1) \quad \text { and }\left[\begin{array}{l}
a \\
b \\
c \\
d \\
e
\end{array}\right]=\left[G^{-1}\right]\left[\begin{array}{l}
d 1 \\
q 1 \\
d 3 \\
q 3
\end{array}\right] . . \text { (2) Where }} \\
& \mathrm{G}=\frac{2}{5}\left[\begin{array}{ccccc}
\sin (\theta) & \sin \left(\theta-72^{\circ}\right) & \sin \left(\theta-144^{\circ}\right) & \sin \left(\theta-216^{\circ}\right) & \sin \left(\theta-288^{\circ}\right) \\
\cos (\theta) & \cos \left(\theta-72^{\circ}\right) & \cos \left(\theta-144^{\circ}\right) & \cos \left(\theta-216^{\circ}\right) & \cos \left(\theta-288^{\circ}\right) \\
\sin (3 \theta) & \sin \left(3\left(\theta-72^{\circ}\right)\right) & \sin \left(3\left(\theta-144^{\circ}\right)\right) & \sin \left(3\left(\theta-216^{\circ}\right)\right) & \sin \left(3\left(\theta-288^{\circ}\right)\right) \\
\cos (3 \theta) & \cos \left(3\left(\theta-72^{\circ}\right)\right) & \cos \left(3\left(\theta-144^{\circ}\right)\right) & \cos \left(3\left(\theta-216^{\circ}\right)\right) & \cos \left(3\left(\theta-288^{\circ}\right)\right)
\end{array}\right] \text { (3) }
\end{aligned}
$$

As the two planes d1-q1 and d3-q3 are orthogonal, the fundamental and the third harmonic components of the voltage can be controlled independently. Also the reference voltage can be mapped into two orthogonal stationary frames $\alpha 1-\beta 1$ and $\alpha 3-\beta 3$ according to $(4,5,6)$.

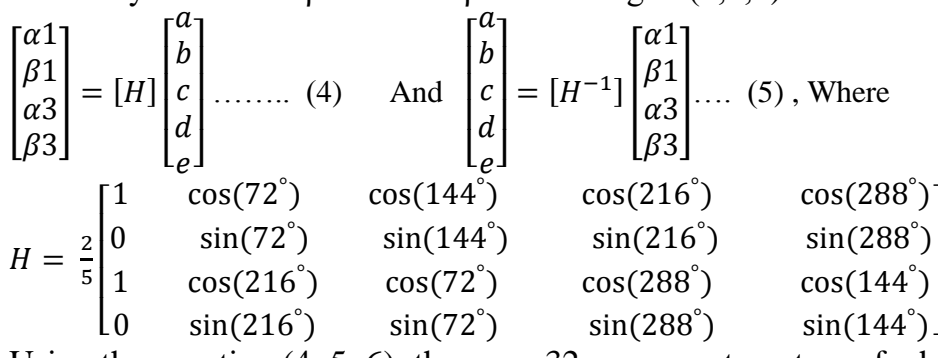

Using the equation $(4,5,6)$ there are 32 space vectors, two of which are zero vectors $(00000$ and 11111$)$. Thirty non-zero space voltage vectors of a five-phase inverter can be projected to $\alpha 1-\beta 1$ plane and $\alpha 3-\beta 3$ palne as shown in Figure 2. In $\alpha 1-\beta 1$ palne, the thirty vectors are composed of three sets of different amplitude vectors, and divide $\alpha 1-\beta 1$ plane into 10 sectors. The amplitudes of these voltage vectors are [312, 15]:-

Vmin = 0.2472Vdc, (11001), (11000), (11100), (01100), (01110), (00110), (00111), (00011), (10011), (10001).

Vmid = 0.4Vdc, (10000), (11101), (01000), (11110), (00100),(01111), (00010), (10111), (00001), (11011). $\mathrm{V} \max =0.6472 \mathrm{Vdc},(01001),(11010),(10100),(01101),(01010),(10110),(00101),(01011),(10010)$, (10101). And the ratio of the amplitudes is 1:1.618:1.6182. It is the same situation in the $\alpha 3-\beta 3$ plane.
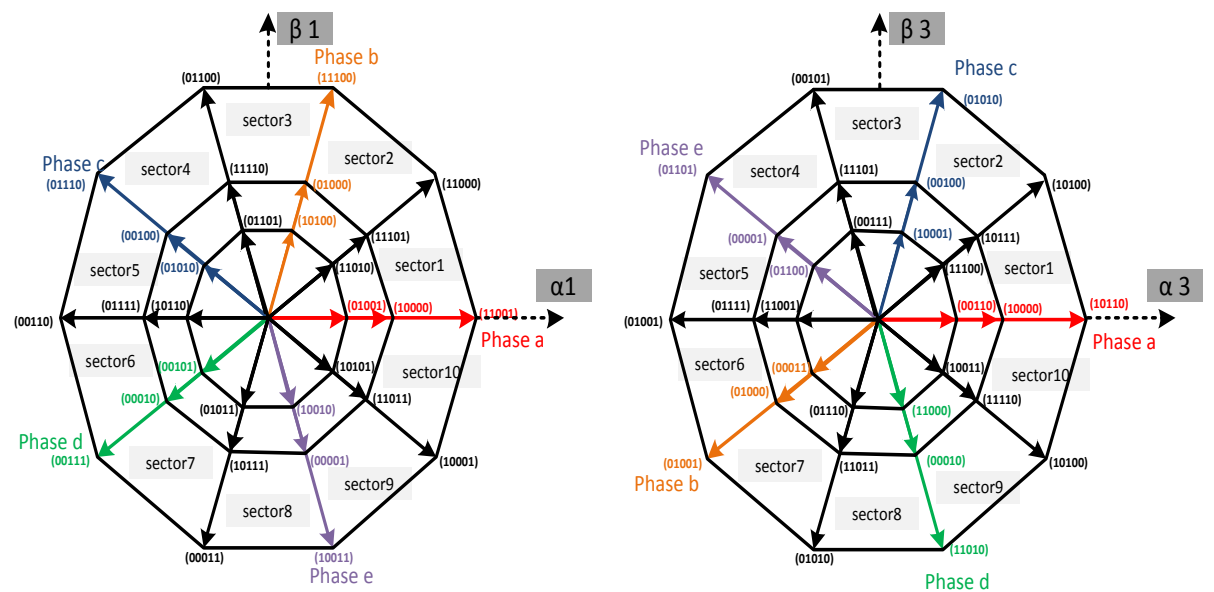

Figure 2. Thirty nonzero switching vectors on $\alpha 1-\beta 1$ plane and $\alpha 3-\beta 3$ plane.

From the average vector concept during one sampling period [12], a reference voltage vector on the $\alpha 1-\beta 1$ plane can be realized by adjusting the application times of the nearest two Vmid switching vectors and two 
Vmax switching vectors. The other combinations of switching vectors increase the number of switching or decrease the maximum magnitude of the realizable voltage vector.

\subsubsection{Calculation of the Application Times for the Switching Vectors}

Figure 3 shows the reference voltage of the fundamental component (Vref_ $\alpha 1-\beta 1$ ) exists in the first quadrant in the $\alpha 1-\beta 1$ plane and the third harmonic component of the reference voltage (Vref_ $\alpha 3-\beta 3$ ) located in the first sector in the $\alpha 3-\beta 3$ plan. For both reference voltages, the vectors $(00000,10000,11000$, $01001,11101,11111)$ are used to utilize them in both $\alpha 1-\beta 1$ and $\alpha 3-\beta 3$ planes as shown in figure 3 .
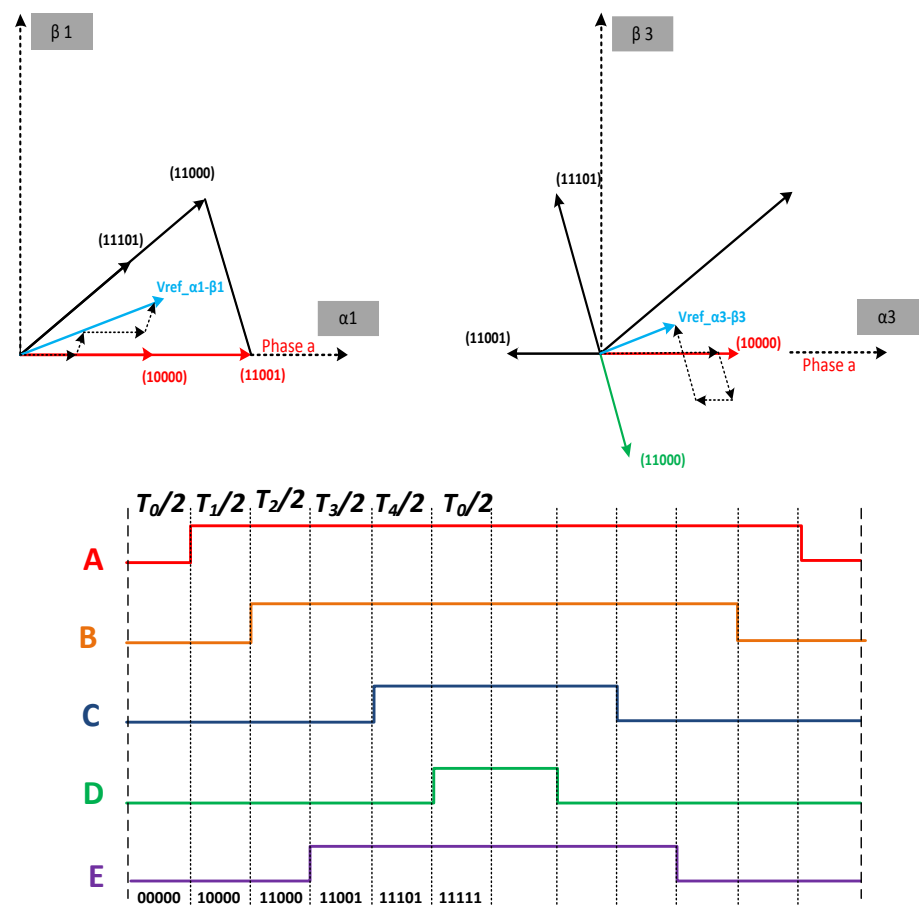

Figure 3. Realization of a reference voltage vector located in sector 1 on the $d-q$ plane and the associated switching sequence

The time needed for applying each vectors can be calculated as:-

$\left[\begin{array}{l}T_{1} \\ T_{2} \\ T_{3} \\ T_{4}\end{array}\right]=T s \times\left[T^{-1}\right]\left[\begin{array}{l}\text { Vref_ } \alpha 1 \\ \text { Vref_} \beta 1 \\ \text { Vref_ } \alpha 3 \\ \text { Vref_ } \beta 3\end{array}\right] \ldots \ldots$ (7) Where

$\mathrm{T}=\left[\begin{array}{cccc}V_{\text {mid }} & V_{\text {max }} \cos \left(72^{\circ}\right) & V_{\text {max }} & V_{\text {mid }} \cos \left(72^{\circ}\right) \\ 0 & V_{\text {max }} \sin \left(72^{\circ}\right) & 0 & V_{\text {mid }} \sin \left(72^{\circ}\right) \\ V_{\text {mid }} & V_{\text {min }} \cos \left(288^{\circ}\right) & -V_{\text {min }} & V_{\text {mid }} \cos \left(108^{\circ}\right) \\ 0 & V_{\text {min }} \sin \left(288^{\circ}\right) & 0 & V_{\text {mid }} \sin \left(108^{\circ}\right)\end{array}\right]$

$T_{0}=T_{s}-T_{1}-T_{2}-T_{3}-T_{4}$

The same approach can be applied for other sectors.

\subsection{Algorithm for tracking the saturation saliency of five phase PMSM}

The stator windings self-inductances are modulated by the anisotropy obtained by the saturation saliency of main flux as shown in [20]. This modulation will be reflected in the transient response of the motor line currents to the test vector imposed by the inverter. So by using the fundamental PWM wave form and by measuring the transient current response to the active vectors, it is possible to detect the inductance variation and track the rotor position. It is worth to say here that the output PWM waveform obtained using the multidimension SVPWM in this work has four active vectors V1,V2,V3, and V4 in addition to the zero vector V0. This number of active vectors make it possible to obtain more than one algorithm to track the saturation saliency. This paper introduces the following algorithms:

\subsubsection{Tracking saliency using the active vectors V1,V4 and V0}

Figure 4 shows the space vector modulation state diagram for a five-phase inverter when V_ref_ $\alpha 1-\beta 1$ exists in first sector. The switching sequences and the timing of the applied vectors is shown in Fig 4. 


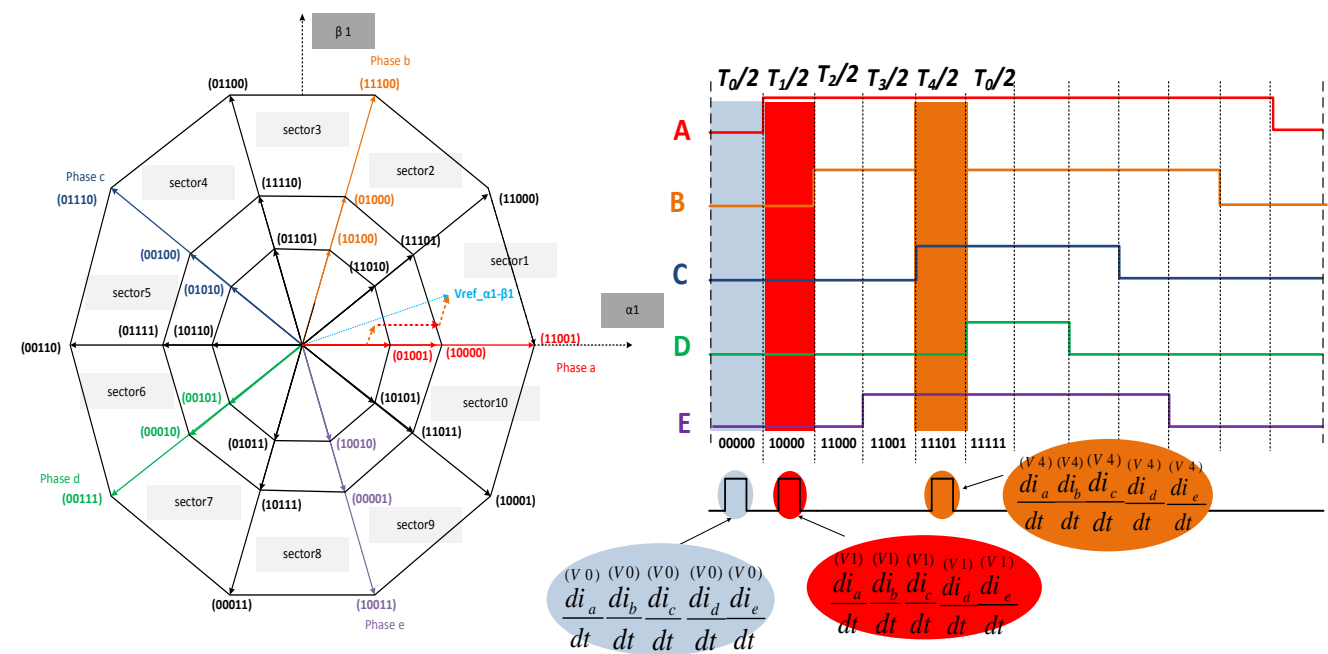

Figure 4. Sampling time of the currents responses due to the switching actions of active vector V1 and V4 in case that V_ref_ $\alpha 1-\beta 1$ exists in first sector

The stator circuit when the vectors V0,V1 and V4 are applied are shown in Figure 5.a, figure 5.b and figure 5.c respectively.
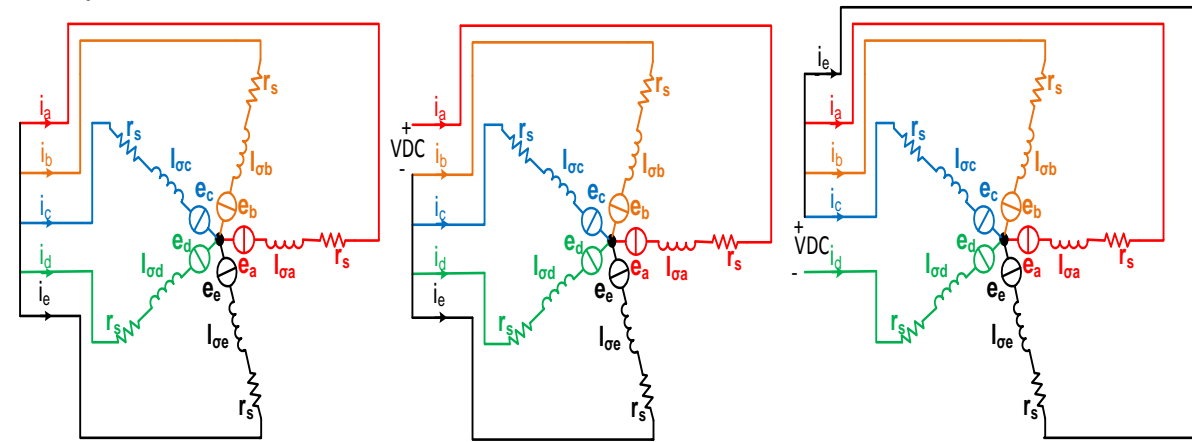

Figure 5. Stator circuits when: (a) V0 is applied; (b) V1 is applied; (c) V4 is applied Using the circuit in Figure 5.a, the following equations hold true:-

$0=r_{s} * i_{a}{ }^{(V 0)}+l_{\sigma a} * \frac{d i_{a}^{(V 0)}}{d t}+e_{a}^{(V 0)}-r_{s} * i_{b}{ }^{(V 0)}-l_{\sigma b} * \frac{d i_{b}^{(V 0)}}{d t}-e_{b}^{(V 0)}$
$0=r_{s} * i_{b}{ }^{(V 0)}+l_{\sigma b} * \frac{d i_{b}^{(V 0)}}{d t}+e_{b}^{(V 0)}-r_{s} * i_{c}{ }^{(V 0)}-l_{\sigma c} * \frac{d i_{c}^{(V 0)}}{d t}-e_{c}^{(V 0)} \ldots$
$0=r_{s} * i_{c}^{(V 0)}+l_{\sigma c} * \frac{d i_{c}^{(V 0)}}{d t}+e_{c}^{(V 0)}-r_{s} * i_{d}^{(V 0)}-l_{\sigma d} * \frac{d i_{d}^{(V 0)}}{d t}-e_{d}^{(V 0)} \ldots$
$0=r_{s} * i_{d}{ }^{(V 0)}+l_{\sigma d} * \frac{d i_{d}^{(V 0)}}{d t}+e_{d}^{(V 0)}-r_{s} * i_{e}{ }^{(V 0)}-l_{\sigma e} * \frac{d i_{e}^{(V 0)}}{d t}-e_{e}^{(V 0)} \ldots$
$0=r_{s} * i_{e}{ }^{(V 0)}+l_{\sigma e} * \frac{d i_{e}^{(V 0)}}{d t}+e_{e}^{(V 0)}-r_{s} * i_{a}{ }^{(V 0)}-l_{\sigma a} * \frac{d i_{a}^{(V 0)}}{d t}-e_{a}^{(V 0)} \ldots$

The following equations are obtained using Figure 5.b:-

$\mathrm{V}_{D C}=r_{s} * i_{a}^{(V 1)}+l_{\sigma a} * \frac{d i_{a}^{(V 1)}}{d t}+e_{a}^{(V 1)}-r_{s} * i_{b}^{(V 1)}-l_{\sigma b} * \frac{d i_{b}^{(V 1)}}{d t}-e_{b}^{(V 1)}$.

$0=r_{s} * i_{b}^{(V 1)}+l_{\sigma b} * \frac{d i_{b}^{(V 1)}}{d t}+e_{b}^{(V 1)}-r_{s} * i_{c}^{(V 1)}-l_{\sigma c} * \frac{d i_{c}^{(V 1)}}{d t}-e_{c}^{(V 1)}$

$0=r_{s} * i_{c}^{(V 1)}+l_{\sigma c} * \frac{d i_{c}^{(V 1)}}{d t}+e_{c}^{(V 1)}-r_{s} * i_{d}^{(V 1)}-l_{\sigma d} * \frac{d i_{d}^{(V 1)}}{d t}-e_{d}^{(V 1)}$

$0=r_{s} * i_{d}^{(V 1)}+l_{\sigma d} * \frac{d i_{d}^{(V 1)}}{d t}+e_{d}^{(V 1)}-r_{s} * i_{e}^{(V 1)}+l_{\sigma e} * \frac{d i_{e}^{(V 1)}}{d t}-e_{e}^{(V 1)}$

$-\mathrm{V}_{D C}=r_{s} * i_{e}^{(V 1)}+l_{\sigma e} * \frac{d i_{e}^{(V 1)}}{d t}+e_{e}^{(V 1)}-r_{s} * i_{a}^{(V 1)}-l_{\sigma a} * \frac{d i_{a}^{(V 1)}}{d t}-e_{a}^{(V 1)}$.

Finally when V4 is applied as shown in Figure 5.c, the following equations hold true:-

$0=r_{s} * i_{a}{ }^{(V 4)}+l_{\sigma a} * \frac{d i_{a}^{(V 4)}}{d t}+e_{a}^{(V 4)}-r_{s} * i_{b}{ }^{(V 4)}-l_{\sigma b} * \frac{d i_{b}^{(V 4)}}{d t}-e_{b}^{(V 4)}$
$0=r_{s} * i_{b}{ }^{(V 4)}+l_{\sigma b} * \frac{d i_{b}^{(V 4)}}{d t}+e_{b}^{(V 4)}-r_{s} * i_{c}{ }^{(V 4)}-l_{\sigma c} * \frac{d i_{c}^{(V 4)}}{d t}-e_{c}^{(V 4)}$ 


$$
\begin{aligned}
& \mathrm{V}_{D C}=r_{s} * i_{c}^{(V 4)}+l_{\sigma c} * \frac{d i_{c}^{(V 4)}}{d t}+e_{c}^{(V 4)}-r_{s} * i_{d}^{(V 4)}-l_{\sigma d} * \frac{d i_{d}^{(V 4)}}{d t}-e_{d}^{(V 4)} \\
& -\mathrm{V}_{D C}=r_{s} * i_{d}^{(V 4)}+l_{\sigma d} * \frac{d i_{d}^{(V 4)}}{d t}+e_{d}^{(V 4)}-r_{s} * i_{e}^{(V 4)}-l_{\sigma e} * \frac{d i_{e}^{(V 4)}}{d t}-e_{e}^{(V 4)} \ldots \\
& 0=r_{s} * i_{e}{ }^{(V 4)}+l_{\sigma e} * \frac{d i_{e}^{(V 4)}}{d t}+e_{e}^{(V 4)}-r_{s} * i_{a}{ }^{(V 4)}-l_{\sigma a} * \frac{d i_{a}^{(V 4)}}{d t}-e_{a}^{(V 4)}
\end{aligned}
$$

Assuming that the voltage drop across the stator resistances are small and the back emf can be cancelled if the time separation between the vectors is small, the position scalars $\mathrm{Pa}, \mathrm{Pb}, \mathrm{Pc}, \mathrm{Pd}$ and $\mathrm{Pe}$ in all sectors are given in table 1 .

\begin{tabular}{|c|c|c|c|c|c|}
\hline Sector no & $\mathrm{Pa}_{\mathrm{a}}$ & $\mathrm{Pb}_{\mathrm{b}}$ & $P_{c}$ & $\mathrm{P}_{\mathrm{d}}$ & $\mathrm{Pe}_{\mathrm{e}}$ \\
\hline 1 & $\frac{d i_{c}^{(V 0)}}{d t}-\frac{d i_{c}^{(V 4)}}{d t}$ & $\frac{d i_{e}^{(V 0)}}{d t}-\frac{d i_{e}^{(V 4)}}{d t}$ & $\frac{d i_{e}^{(V 1)}}{d t}-\frac{d i_{e}^{(V 0)}}{d t}$ & $\frac{d i_{b}^{(V 1)}}{d t}-\frac{d i_{b}^{(V 0)}}{d t}$ & $-\left(\mathrm{P}_{\mathrm{a}}+\mathrm{P}_{\mathrm{b}}+\mathrm{P}_{\mathrm{c}}+\mathrm{P}_{\mathrm{d}}\right)$ \\
\hline 2 & $\frac{d i_{c}^{(V 0)}}{d t}-\frac{d i_{c}^{(V 4)}}{d t}$ & $\frac{d i_{e}^{(V 0)}}{d t}-\frac{d i_{e}^{(V 4)}}{d t}$ & $-\left(\mathrm{P}_{\mathrm{a}}+\mathrm{P}_{\mathrm{b}}+\mathrm{P}_{\mathrm{d}}+\mathrm{Pe}_{\mathrm{e}}\right)$ & $\frac{d i_{a}^{(V 1)}}{d t}-\frac{d i_{a}^{(V 0)}}{d t}$ & $\frac{d i_{c}^{(V 1)}}{d t}-\frac{d i_{a c}^{(V 0)}}{d t}$ \\
\hline 3 & $-\left(\mathrm{P}_{\mathrm{b}}+\mathrm{P}_{\mathrm{c}}+\mathrm{P}_{\mathrm{d}}+\mathrm{P}_{\mathrm{e}}\right)$ & $\frac{d i_{d}^{(V 0)}}{d t}-\frac{d i_{d}^{(V 4)}}{d t}$ & $\frac{d i_{a}^{(V 0)}}{d t}-\frac{d i_{a}^{(V 4)}}{d t}$ & $\frac{d i_{a}^{(V 1)}}{d t}-\frac{d i_{a}^{(V 0)}}{d t}$ & $\frac{d i_{c}^{(V 1)}}{d t}-\frac{d i_{c}^{(V 0)}}{d t}$ \\
\hline 4 & $\frac{d i_{d}^{(V 1)}}{d t}-\frac{d i_{d}^{(V 0)}}{d t}$ & $\frac{d i_{d}^{(V 0)}}{d t}-\frac{d i_{d}^{(V 4)}}{d t}$ & $\frac{d i_{a}^{(V 0)}}{d t}-\frac{d i_{a}^{(V 4)}}{d t}$ & $-(\mathrm{Pa}+\mathrm{Pb}+\mathrm{Pc}+\mathrm{Pe})$ & $\frac{d i_{b}^{(V 1)}}{d t}-\frac{d i_{a b}^{(V 0)}}{d t}$ \\
\hline 5 & $\frac{d i_{d}^{(V 1)}}{d t}-\frac{d i_{d}^{(V 0)}}{d t}$ & $-\left(\mathrm{P}_{\mathrm{a}}+\mathrm{P}_{\mathrm{c}}+\mathrm{P}_{\mathrm{d}}+\mathrm{P}_{\mathrm{e}}\right)$ & $\frac{d i_{e}^{(V 0)}}{d t}-\frac{d i_{e}^{(V 4)}}{d t}$ & $\frac{d i_{b}^{(V 0)}}{d t}-\frac{d i_{b}^{(V 4)}}{d t}$ & $\frac{d i_{b}^{(V 1)}}{d t}-\frac{d i_{a b}^{(V 0)}}{d t}$ \\
\hline 6 & $\frac{d i_{c}^{(V 1)}}{d t}-\frac{d i_{c}^{(V 0)}}{d t}$ & $\frac{d i_{e}^{(V 1)}}{d t}-\frac{d i_{e}^{(V 0)}}{d t}$ & $\frac{d i_{e}^{(V 0)}}{d t}-\frac{d i_{e}^{(V 4)}}{d t}$ & $\frac{d i_{b}^{(V 0)}}{d t}-\frac{d i_{b}^{(V 4)}}{d t}$ & $-\left(\mathrm{P}_{\mathrm{a}}+\mathrm{P}_{\mathrm{b}}+\mathrm{P}_{\mathrm{c}}+\mathrm{P}_{\mathrm{d}}\right)$ \\
\hline 7 & $\frac{d i_{c}^{(V 1)}}{d t}-\frac{d i_{c}^{(V 0)}}{d t}$ & $\frac{d i_{e}^{(V 1)}}{d t}-\frac{d i_{e}^{(V 0)}}{d t}$ & $-\left(\mathrm{P}_{\mathrm{a}}+\mathrm{P}_{\mathrm{b}}+\mathrm{P}_{\mathrm{d}}+\mathrm{P}_{\mathrm{e}}\right)$ & $\frac{d i_{a}^{(V 0)}}{d t}-\frac{d i_{a}^{(V 4)}}{d t}$ & $\frac{d i_{c}^{(V 0)}}{d t}-\frac{d i_{c}^{(V 4)}}{d t}$ \\
\hline 8 & $-\left(\mathrm{P}_{\mathrm{b}}+\mathrm{P}_{\mathrm{c}}+\mathrm{P}_{\mathrm{d}}+\mathrm{P}_{\mathrm{e}}\right)$ & $\frac{d i_{d}^{(V 1)}}{d t}-\frac{d i_{d}^{(V 0)}}{d t}$ & $\frac{d i_{a}^{(V 1)}}{d t}-\frac{d i_{a}^{(V 0)}}{d t}$ & $\frac{d i_{a}^{(V 0)}}{d t}-\frac{d i_{a}^{(V 4)}}{d t}$ & $\frac{d i_{c}^{(V 0)}}{d t}-\frac{d i_{c}^{(V 4)}}{d t}$ \\
\hline 9 & $\frac{d i_{d}^{(V 0)}}{d t}-\frac{d i_{d}^{(V 4)}}{d t}$ & $\frac{d i_{d}^{(V 1)}}{d t}-\frac{d i_{d}^{(V 0)}}{d t}$ & $\frac{d i_{a}^{(V 1)}}{d t}-\frac{d i_{a}^{(V 0)}}{d t}$ & $-\left(\mathrm{P}_{\mathrm{a}}+\mathrm{P}_{\mathrm{b}}+\mathrm{P}_{\mathrm{c}}+\mathrm{P}_{\mathrm{e}}\right)$ & $\frac{d i_{b}^{(V 0)}}{d t}-\frac{d i_{b}^{(V 4)}}{d t}$ \\
\hline 10 & $\frac{d i_{d}^{(V 0)}}{d t}-\frac{d i_{d}^{(V 4)}}{d t}$ & $-(\mathrm{Pa}+\mathrm{Pc}+\mathrm{Pd}+\mathrm{Pe})$ & $\frac{d i_{e}^{(V 1)}}{d t}-\frac{d i_{e}^{(V 0)}}{d t}$ & $\frac{d i_{b}^{(V 1)}}{d t}-\frac{d i_{b}^{(V 0)}}{d t}$ & $\frac{d i_{b}^{(V 0)}}{d t}-\frac{d i_{b}^{(V 4)}}{d t}$ \\
\hline
\end{tabular}

Table1 Selection of $\mathrm{Pa}, \mathrm{Pb}, \mathrm{Pc}, \mathrm{Pd}$ and $\mathrm{Pe}$ for a star-connected five machine by sampling switching actions of active vector V1 and V4

\subsubsection{Tracking saliency using the vectors $\mathrm{V} 2, \mathrm{~V} 3$ and $\mathrm{V0}$}

Figure 6 shows the space vector modulation state diagram for a five-phase inverter when V_ref_ $\alpha 1-\beta 1$ exists in first sector. The switching sequences and the timing of the applied vectors will be :-

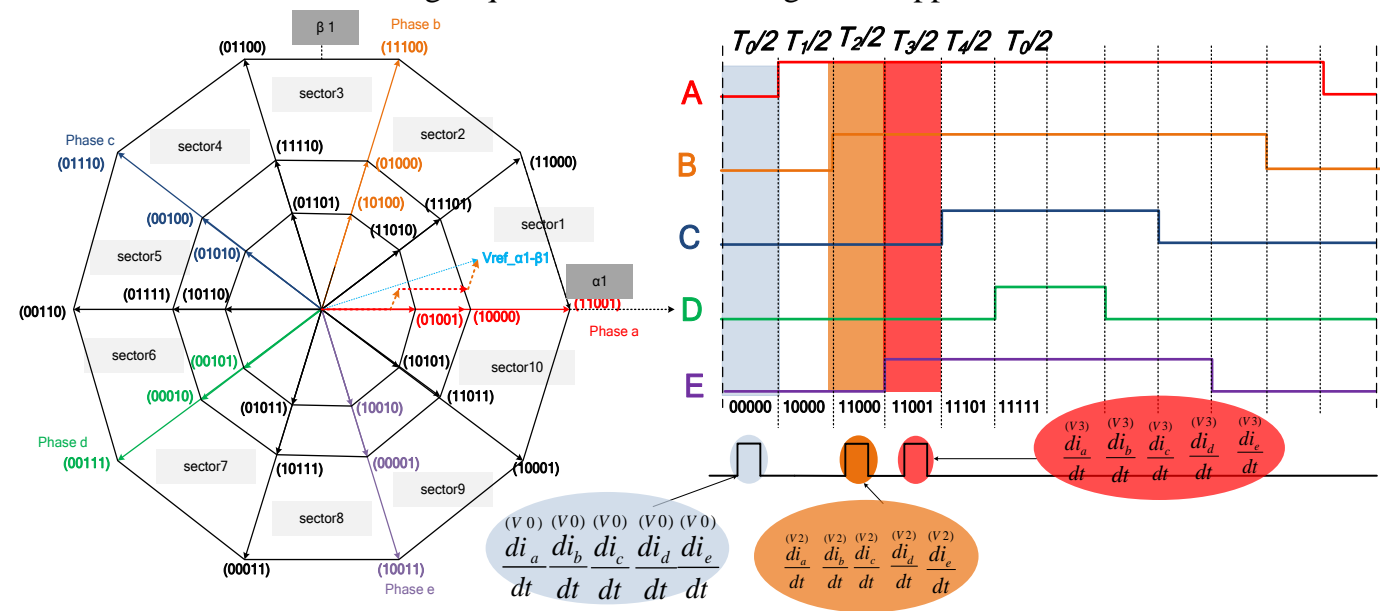

Figure 6. Sampling time of the currents responses due to the switching actions of active vector V2 and V3 in case that V_ref_ $\alpha 1-\beta 1$ exists in first sector 
The stator circuit when the vectors V0,V2 and V3 are applied are shown in Figure 7.a, figure 7.b and figure 7.c respectively.

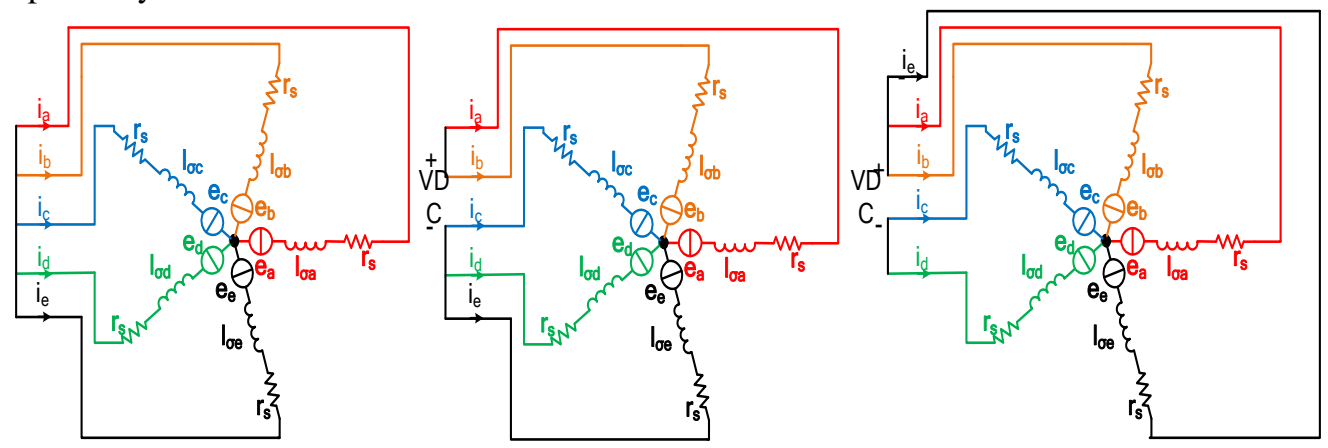

Figure 7. Stator circuits when: (a) V0 is applied; (b) V2 is applied; (c) V3 is applied

Using the circuit in Figure 7.a, the following equations hold true:-

$0=r_{s} * i_{a}{ }^{(V 0)}+l_{\sigma a} * \frac{d i_{a}^{(V 0)}}{d t}+e_{a}^{(V 0)}-r_{s} * i_{b}{ }^{(V 0)}-l_{\sigma b} * \frac{d i_{b}^{(V 0)}}{d t}-e_{b}^{(V 0)}$
$0=r_{s} * i_{b}{ }^{(V 0)}+l_{\sigma b} * \frac{d i_{b}^{(V 0)}}{d t}+e_{b}^{(V 0)}-r_{s} * i_{c}{ }^{(V 0)}-l_{\sigma c} * \frac{d i_{c}{ }^{(V 0)}}{d t}-e_{c}^{(V 0)}$
$0=r_{s} * i_{c}{ }^{(V 0)}+l_{\sigma c} * \frac{d i_{c}^{(V 0)}}{d t}+e_{c}^{(V 0)}-r_{s} * i_{d}^{(V 0)}-l_{\sigma d} * \frac{d i_{d}^{(V 0)}}{d t}-e_{d}^{(V 0)}$
$0=r_{s} * i_{d}{ }^{(V 0)}+l_{\sigma d} * \frac{d i_{d}^{(V 0)}}{d t}+e_{d}^{(V 0)}-r_{s} * i_{e}{ }^{(V 0)}-l_{\sigma e} * \frac{d i_{e}^{(V 0)}}{d t}-e_{e}^{(V 0)}$
$0=r_{s} * i_{e}{ }^{(V 0)}+l_{\sigma e} * \frac{d i_{e}^{(V 0)}}{d t}+e_{e}^{(V 0)}-r_{s} * i_{a}{ }^{(V 0)}-l_{\sigma a} * \frac{d i_{a}^{(V 0)}}{d t}-e_{a}^{(V 0)}$

The following equations are obtained using Figure 7.b:-

$0=r_{s} * i_{a}^{(V 2)}+l_{\sigma a} * \frac{d i_{a}^{(V 2)}}{d t}+e_{a}^{(V 1)}-r_{s} * i_{b}^{(V 2)}-l_{\sigma b} * \frac{d i_{b}^{(V 2)}}{d t}-e_{b}^{(V 2)}$.

$\mathrm{V}_{D C}=r_{s} * i_{b}^{(V 2)}+l_{\sigma b} * \frac{d i_{b}^{(V 2)}}{d t}+e_{b}^{(V 2)}-r_{s} * i_{c}^{(V 2)}-l_{\sigma c} * \frac{d i_{c}^{(V 2)}}{d t}-e_{c}^{(V 2)}$

$0=r_{s} * i_{c}^{(V 2)}+l_{\sigma c} * \frac{d i_{c}^{(V 2)}}{d t}+e_{c}^{(V 2)}-r_{s} * i_{d}^{(V 2)}-l_{\sigma d} * \frac{d i_{d}^{(V 2)}}{d t}-e_{d}^{(V 2)}$

$0=r_{s} * i_{d}^{(V 2)}+l_{\sigma d} * \frac{d i_{d}^{(V 2)}}{d t}+e_{d}^{(V 2)}-r_{s} * i_{e}^{(V 2)}+l_{\sigma e} * \frac{d i_{e}^{(V 2)}}{d t}-e_{e}^{(V 2)}$

$-\mathrm{V}_{D C}=r_{s} * i_{e}^{(V 2)}+l_{\sigma e} * \frac{d i_{e}^{(V 2)}}{d t}+e_{e}^{(V 2)}-r_{s} * i_{a}^{(V 2)}-l_{\sigma a} * \frac{d i_{a}^{(V 2)}}{d t}-e_{a}^{(V 2)}$.

Finally when V3 is applied as shown in Figure 7.c, the following equations hold true:-

$0=r_{s} * i_{a}{ }^{(V 3)}+l_{\sigma a} * \frac{d i_{a}^{(V 3)}}{d t}+e_{a}^{(V 3)}-r_{s} * i_{b}{ }^{(V 3)}-l_{\sigma b} * \frac{d i_{b}^{(V 3)}}{d t}-e_{b}^{(V 3)}$

$\mathrm{V}_{D C}=r_{s} * i_{b}{ }^{(V 3)}+l_{\sigma b} * \frac{d i_{b}^{(V 4)}}{d t}+e_{b}^{(V 3)}-r_{s} * i_{c}{ }^{(V 3)}-l_{\sigma c} * \frac{d i_{c}^{(V 3)}}{d t}-e_{c}^{(V 3)}$

$0=r_{s} * i_{c}{ }^{(V 3)}+l_{\sigma c} * \frac{d i_{c}^{(V 3)}}{d t}+e_{c}^{(V 3)}-r_{s} * i_{d}{ }^{(V 3)}-l_{\sigma d} * \frac{d i_{d}^{(V 3)}}{d t}-e_{d}^{(V 3)}$

$-\mathrm{V}_{D C}=r_{s} * i_{d}^{(V 3)}+l_{\sigma d} * \frac{d i_{d}^{(V 3)}}{d t}+e_{d}^{(V 3)}-r_{s} * i_{e}^{(V 3)}-l_{\sigma e} * \frac{d i_{e}^{(V 3)}}{d t}-e_{e}^{(V 3)}$.

$0=r_{s} * i_{e}^{(V 3)}+l_{\sigma e} * \frac{d i_{e}^{(V 3)}}{d t}+e_{e}^{(V 3)}-r_{s} * i_{a}^{(V 3)}-l_{\sigma a} * \frac{d i_{a}^{(V 3)}}{d t}-e_{a}^{(V 3)}$

Assuming that the voltage drop across the stator resistances are small and the back emf can be cancelled if the time separation between the vectors is small, the position scalars $\mathrm{Pa}, \mathrm{Pb}, \mathrm{Pc}, \mathrm{Pd}$ and $\mathrm{Pe}$ in all sectors are given in table 2 .

The position scalars $\mathrm{Pa}, \mathrm{Pb}, \mathrm{Pc}, \mathrm{Pd}$ and $\mathrm{Pe}$ either obtained from table 1 or table 2 can be transformed into $P_{\alpha}, P_{\beta}$ and can then be used to denote the orientation angle as follows :-

$\left[\begin{array}{l}P_{\alpha} \\ P_{\beta}\end{array}\right]=[V]\left[\begin{array}{l}a \\ b \\ c \\ d \\ e\end{array}\right]\left(\frac{d i_{d}^{(V 4)}}{d t}-\frac{d i_{d}^{(V 0)}}{d t}\right) \ldots \ldots \ldots . .(40), \quad$ Where

$V=\left[\begin{array}{ccccc}1 & \cos \left(216^{\circ}\right) & \cos \left(72^{\circ}\right) & \cos \left(288^{\circ}\right) & \cos \left(144^{\circ}\right) \\ 0 & \sin \left(216^{\circ}\right) & \sin \left(72^{\circ}\right) & \sin (288) & \sin \left(144^{\circ}\right)\end{array}\right]$ 
Table 2 Selection of $\mathrm{Pa}, \mathrm{Pb}, \mathrm{Pc}, \mathrm{Pd}$ and $\mathrm{Pe}$ for a star-connected five machine by sampling switching actions of active vector $\mathrm{V} 2$ and $\mathrm{V} 3$

\begin{tabular}{|c|c|c|c|c|c|}
\hline Sector no & $\mathrm{P}_{\mathrm{a}}$ & $\mathrm{P}_{\mathrm{b}}$ & $\mathrm{P}_{\mathrm{c}}$ & $P_{d}$ & $\mathrm{Pe}_{\mathrm{e}}$ \\
\hline 1 & $\frac{d i_{c}^{(V 0)}}{d t}-\frac{d i_{c}^{(V 2)}}{d t}$ & $\frac{d i_{e}^{(V 0)}}{d t}-\frac{d i_{e}^{(V 2)}}{d t}$ & $\frac{d i_{e}^{(V 3)}}{d t}-\frac{d i_{e}^{(V 0)}}{d t}$ & $\frac{d i_{b}^{(V 3)}}{d t}-\frac{d i_{b}^{(V 0)}}{d t}$ & $-\left(\mathrm{P}_{\mathrm{a}}+\mathrm{P}_{\mathrm{b}}+\mathrm{P}_{\mathrm{c}}+\mathrm{P}_{\mathrm{d}}\right)$ \\
\hline 2 & $\frac{d i_{c}^{(V 0)}}{d t}-\frac{d i_{c}^{(V 2)}}{d t}$ & $\frac{d i_{e}^{(V 0)}}{d t}-\frac{d i_{e}^{(V 2)}}{d t}$ & $-\left(\mathrm{P}_{\mathrm{a}}+\mathrm{P}_{\mathrm{b}}+\mathrm{P}_{\mathrm{d}}+\mathrm{P}_{\mathrm{e}}\right)$ & $\frac{d i_{a}^{(V 3)}}{d t}-\frac{d i_{a}^{(V 0)}}{d t}$ & $\frac{d i_{c}^{(V 3)}}{d t}-\frac{d i_{a c}^{(V 0)}}{d t}$ \\
\hline 3 & $-\left(\mathrm{P}_{b}+\mathrm{P}_{\mathrm{c}}+\mathrm{P}_{\mathrm{d}}+\mathrm{P}_{\mathrm{e}}\right)$ & $\frac{d i_{d}^{(V 0)}}{d t}-\frac{d i_{d}^{(V 2)}}{d t}$ & $\frac{d i_{a}^{(V 0)}}{d t}-\frac{d i_{a}^{(2)}}{d t}$ & $\frac{d i_{a}^{(V 3)}}{d t}-\frac{d i_{a}^{(V 0)}}{d t}$ & $\frac{d i_{c}^{(V 3)}}{d t}-\frac{d i_{c}^{(V 0)}}{d t}$ \\
\hline 4 & $\frac{d i_{d}^{(V 3)}}{d t}-\frac{d i_{d}^{(V 0)}}{d t}$ & $\frac{d i_{d}^{(V 0)}}{d t}-\frac{d i_{d}^{(V 2)}}{d t}$ & $\frac{d i_{a}^{(V 0)}}{d t}-\frac{d i_{a}^{(V 2)}}{d t}$ & $-(\mathrm{Pa}+\mathrm{Pb}+\mathrm{Pc}+\mathrm{Pe})$ & $\frac{d i_{b}^{(V 3)}}{d t}-\frac{d i_{a b}^{(V 0)}}{d t}$ \\
\hline 5 & $\frac{d i_{d}^{(V 3)}}{d t}-\frac{d i_{d}^{(V 0)}}{d t}$ & $-\left(\mathrm{P}_{\mathrm{a}}+\mathrm{P}_{\mathrm{c}}+\mathrm{P}_{\mathrm{d}}+\mathrm{P}_{\mathrm{e}}\right)$ & $\frac{d i_{e}^{(V 0)}}{d t}-\frac{d i_{e}^{(V 2)}}{d t}$ & $\frac{d i_{b}^{(V 0)}}{d t}-\frac{d i_{b}^{(V 2)}}{d t}$ & $\frac{d i_{b}^{(V 3)}}{d t}-\frac{d i_{a b}^{(V 0)}}{d t}$ \\
\hline 6 & $\frac{d i_{c}^{(V 3)}}{d t}-\frac{d i_{c}^{(V 0)}}{d t}$ & $\frac{d i_{e}^{(V 3)}}{d t}-\frac{d i_{e}^{(V 0)}}{d t}$ & $\frac{d i_{e}^{(V 0)}}{d t}-\frac{d i_{e}^{(V 2)}}{d t}$ & $\frac{d i_{b}^{(V 0)}}{d t}-\frac{d i_{b}^{(V 2)}}{d t}$ & $-\left(\mathrm{P}_{\mathrm{a}}+\mathrm{P}_{\mathrm{b}}+\mathrm{P}_{\mathrm{c}}+\mathrm{P}_{\mathrm{d}}\right)$ \\
\hline 7 & $\frac{d i_{c}^{(V 3)}}{d t}-\frac{d i_{c}^{(V 0)}}{d t}$ & $\frac{d i_{e}^{(V 3)}}{d t}-\frac{d i_{e}^{(V 0)}}{d t}$ & $-\left(\mathrm{P}_{\mathrm{a}}+\mathrm{P}_{\mathrm{b}}+\mathrm{P}_{\mathrm{d}}+\mathrm{P}_{\mathrm{e}}\right)$ & $\frac{d i_{a}^{(V 0)}}{d t}-\frac{d i_{a}^{(V 2)}}{d t}$ & $\frac{d i_{c}^{(V 0)}}{d t}-\frac{d i_{c}^{(V 2)}}{d t}$ \\
\hline 8 & $-\left(\mathrm{P}_{b}+\mathrm{P}_{c}+\mathrm{P}_{d}+\mathrm{P}_{\mathrm{e}}\right)$ & $\frac{d i_{d}^{(V 3)}}{d t}-\frac{d i_{d}^{(V 0)}}{d t}$ & $\frac{d i_{a}^{(V 3)}}{d t}-\frac{d i_{a}^{(V 0)}}{d t}$ & $\frac{d i_{a}^{(V 0)}}{d t}-\frac{d i_{a}^{(V 2)}}{d t}$ & $\frac{d i_{c}^{(V 0)}}{d t}-\frac{d i_{c}^{(V 2)}}{d t}$ \\
\hline 9 & $\frac{d i_{d}^{(V 0)}}{d t}-\frac{d i_{d}^{(V 2)}}{d t}$ & $\frac{d i_{d}^{(V 3)}}{d t}-\frac{d i_{d}^{(V 0)}}{d t}$ & $\frac{d i_{a}^{(V 3)}}{d t}-\frac{d i_{a}^{(V 0)}}{d t}$ & $-\left(\mathrm{P}_{\mathrm{a}}+\mathrm{P}_{\mathrm{b}}+\mathrm{P}_{\mathrm{c}}+\mathrm{P}_{\mathrm{e}}\right)$ & $\frac{d i_{b}^{(V 0)}}{d t}-\frac{d i_{b}^{(V 2)}}{d t}$ \\
\hline 10 & $\frac{d i_{d}^{(V 0)}}{d t}-\frac{d i_{d}^{(V 2)}}{d t}$ & $-(\mathrm{Pa}+\mathrm{Pc}+\mathrm{Pd}+\mathrm{Pe})$ & $\frac{d i_{e}^{(V 3)}}{d t}-\frac{d i_{e}^{(V 0)}}{d t}$ & $\frac{d i_{b}^{(V 3)}}{d t}-\frac{d i_{b}^{(V 0)}}{d t}$ & $\frac{d i_{b}^{(V 0)}}{d t}-\frac{d i_{b}^{(V 2)}}{d t}$ \\
\hline
\end{tabular}

3. Results and Analysis

\subsection{Position and Speed Estimation under Sensored Operation}

The validation of the saliency tracking algorithms given in table 1 and table 2 are made using the vector control structure working in sensored mode shown in figure 8. The mechanical observer [23] is used to filter out the high frequency noise in the position signals. The whole vector control structure has been implemented in simulation in the Saber modeling environment. Note the simulation includes a minimum pulse width of 10 us when di/dt measurements are made - a realistic values seen from experimental results of [22].

The results shown in figure 9 demonstrate the validity of the saliency tracking algorithms. The motor was working at $120 \mathrm{rpm}$ speed and at half load. At $\mathrm{t}=2 \mathrm{~s}$ a speed step change from $120 \mathrm{rpm}$ to $0 \mathrm{rpm}$ was applied to the system. Then at $\mathrm{t}=3 \mathrm{~s}$ another speed step change from $0 \mathrm{rpm}$ to $-120 \mathrm{rpm}$ was introduced to the system. After that at $\mathrm{t}=5 \mathrm{~s}$ a zero speed was applied to the system and finally at $\mathrm{t}=6 \mathrm{~s}$ a speed step change from $0 \mathrm{rpm}$ to $120 \mathrm{rpm}$ was applied to the system. The algorithm that is used to track the saliency from the begging to $\mathrm{t}=4 \mathrm{~s}$ is the one given in table 1 (using V1, V4 and V0) while the algorithm given in table 2 (using $\mathrm{V} 2, \mathrm{~V} 3$ and $\mathrm{V} 0$ ) is used to track the saliency for the rest of the test. The results show that the motor responded to the load and speed steps very fast and the proposed algorithms could track the saturation saliency $(2 * \mathrm{fe})$ at positive and negative speeds and more importantly at low and zero speeds. Finally, the position scalars $\mathrm{Pa}, \mathrm{Pb}, \mathrm{Pc}, \mathrm{Pd}$ and $\mathrm{Pe}$ obtained from the algorithm given in table 2 has higher amplitude compared to those obtained from the algorithm given in table 1 and hence it can be said that first advantage of using the algorithm given in table 2 over using the algorithm given in table 1 is the higher signal to noise ratio.

\subsection{Fully Sensorless Speed Control}

The speed control for a five-phase PM machine drive has been implemented in simulation in the Saber modeling environment. This estimated speed $\omega_{r}{ }^{\wedge}$ and position $\theta_{r}{ }^{\wedge}$ are used to obtain a fully sensorless speed control as shown in figure 10 . 


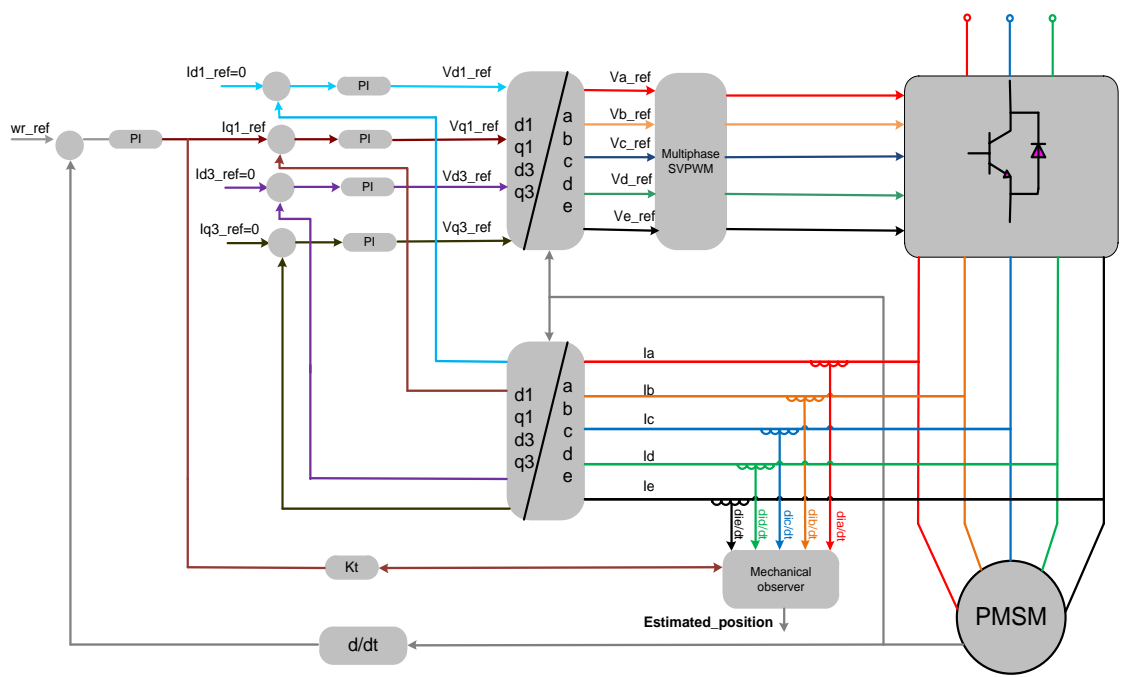

Figure 8 saliency tracking control topology for five phase drive

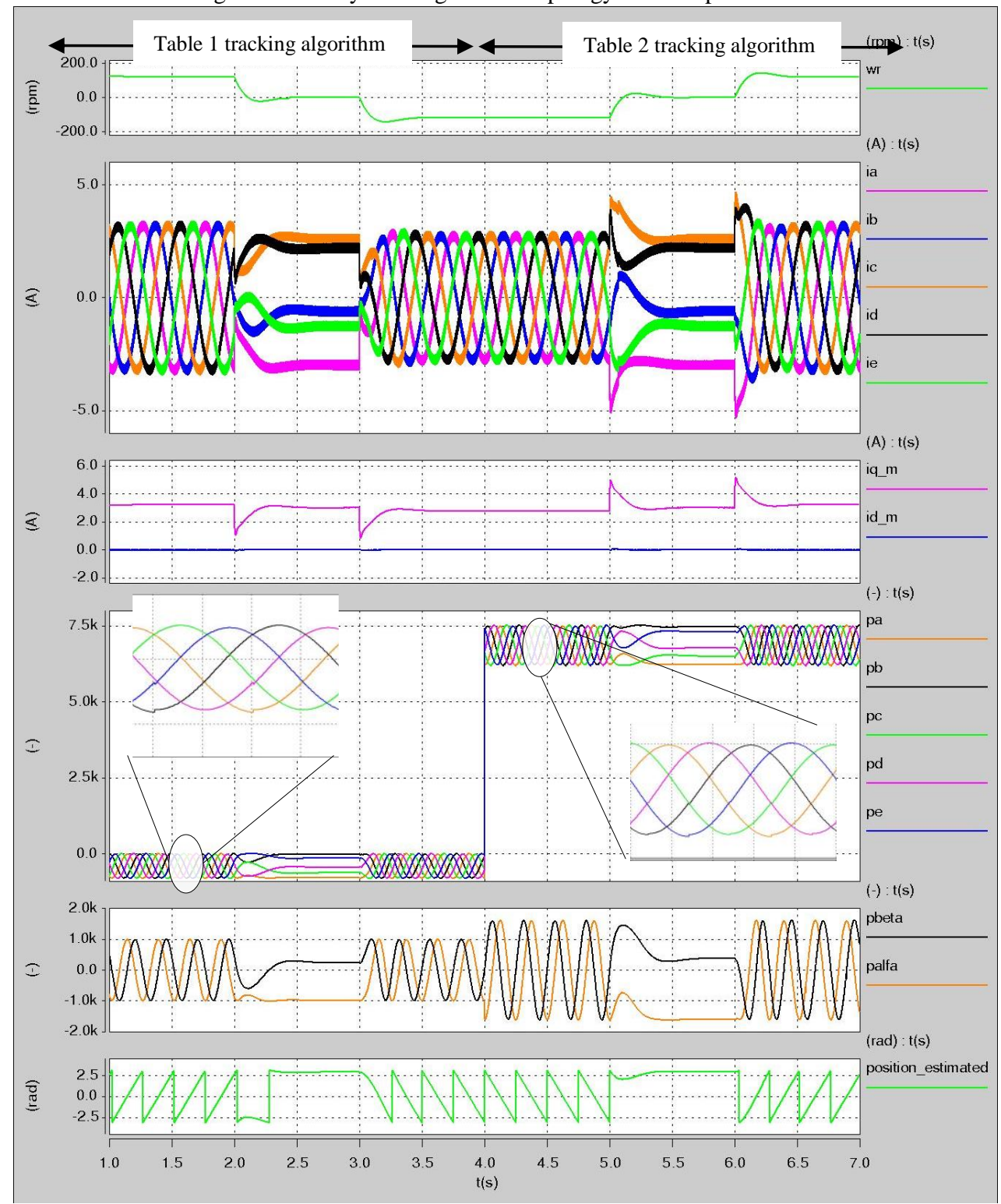

Figur 9 . saliency tracking results 


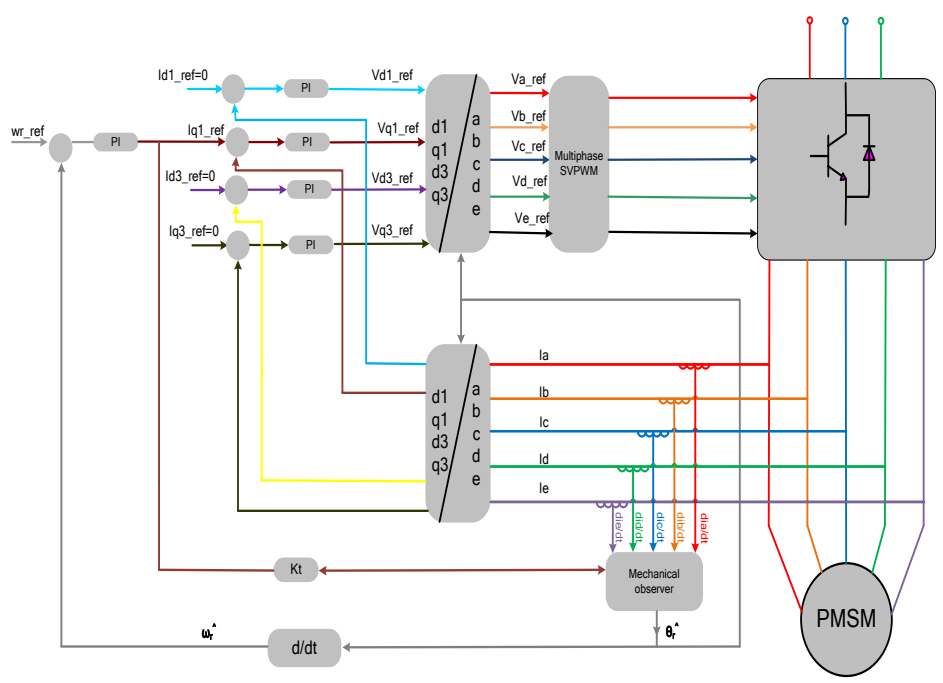

Figure10. the sensorless vector control structure for the five-phase inverter PMSM drive

Figure 11 shows the results of a fully sensorless speed control of a five phase PMSM motor driven by a five phase inverter at a half load condition using the algorithms presented in this paper. The motor was working in sensorless mode at speed $=1 \mathrm{~Hz}$ then at $\mathrm{t}=6 \mathrm{~s}$ a speed step change from $1 \mathrm{~Hz}$ to $0 \mathrm{rpm}$ (till $\mathrm{t}=8$ $\mathrm{s})$ is applied to the system. The algorithm given in table 1 is used to track the saliency from the begging till $\mathrm{t}=7 \mathrm{~s}$ then the algorithm given in table 2 is used for the rest of the test. Figure 11 shows that the motor responded to the speed step with a good transient and steady state response regardless of which algorithm to track the saliency is used.

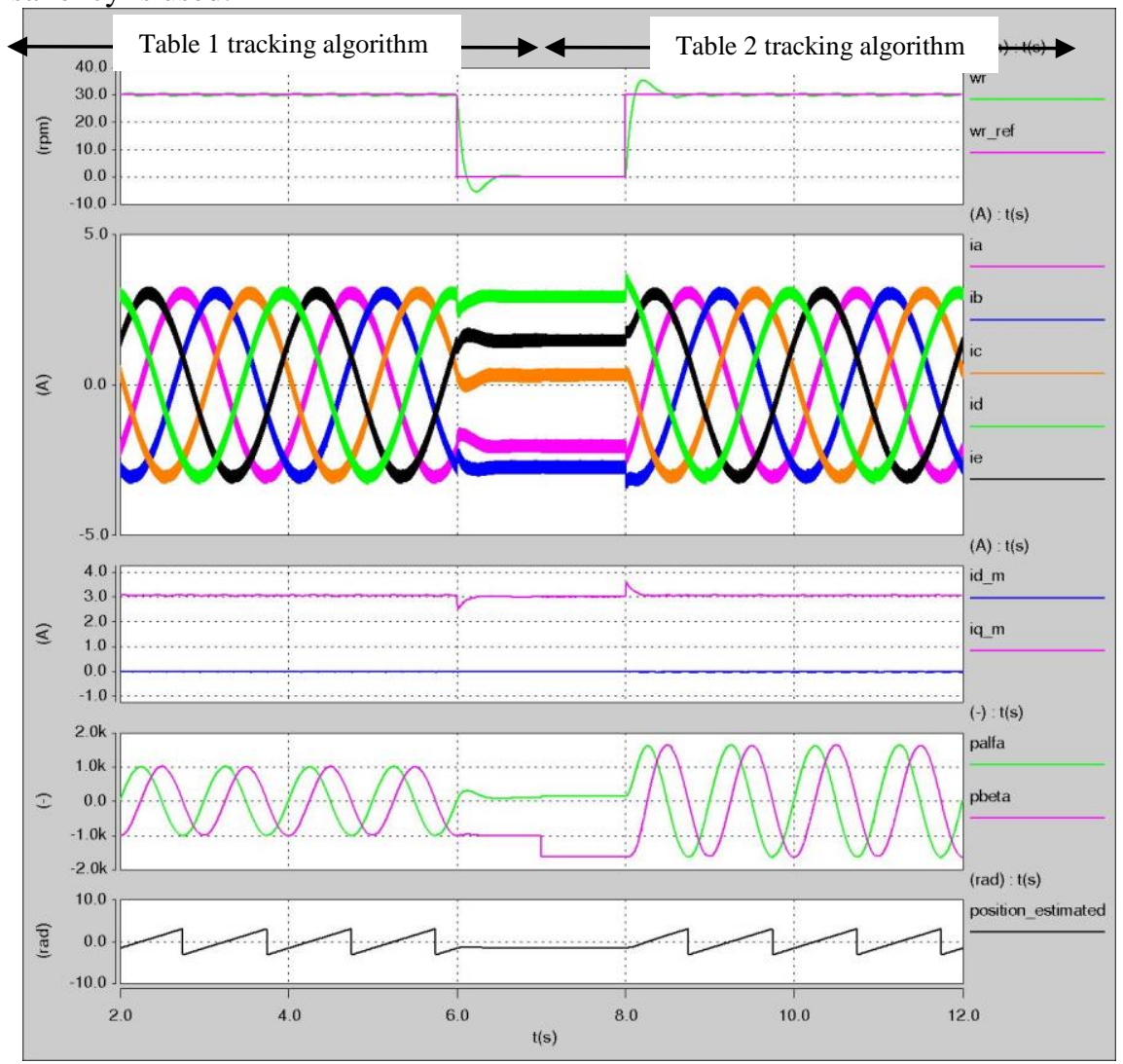

Figure 11 Fully Sensorless Speed Steps between $0.5 \mathrm{~Hz}, 0$ to $0.5 \mathrm{~Hz}$ at half load.

Figure 12 demonstrate the stability of the fully sensorless system when a load disturbance where applied. The motor was working at zero speed and at no load in sensorless mode. Then a step change from $0 \mathrm{rpm}$ to $100 \mathrm{rpm}$ is applied to the system at $\mathrm{t}=2 \mathrm{~s}$. After that a full load step is applied to the motor at $\mathrm{t}=3 \mathrm{~s}$. At $\mathrm{t}=5 \mathrm{~s}$ 
a speed step change from $100 \mathrm{rpm}$ to $0 \mathrm{rpm}$ is applied to the system. Then at $\mathrm{t}=5 \mathrm{~s}$ the motor became unloaded. After the at $\mathrm{t}=7 \mathrm{~s}$ a full load step is applied again to the system. At $\mathrm{t}=8 \mathrm{~s}$ a speed step change from $0 \mathrm{rpm}$ to $100 \mathrm{rpm}$ is applied to the system. After that at $\mathrm{t}=9 \mathrm{~s}$ the motor became unloaded again. Finally at $\mathrm{t}=10 \mathrm{~s}$ a speed step change from $100 \mathrm{rpm}$ to zero is applied to the system. The algorithm given in table 1 is used to track the saliency from the begging till $\mathrm{t}=7 \mathrm{~s}$ then the algorithm given in table 2 is used for the rest of the test. The results shows that the system maintained the speed in all the cases.

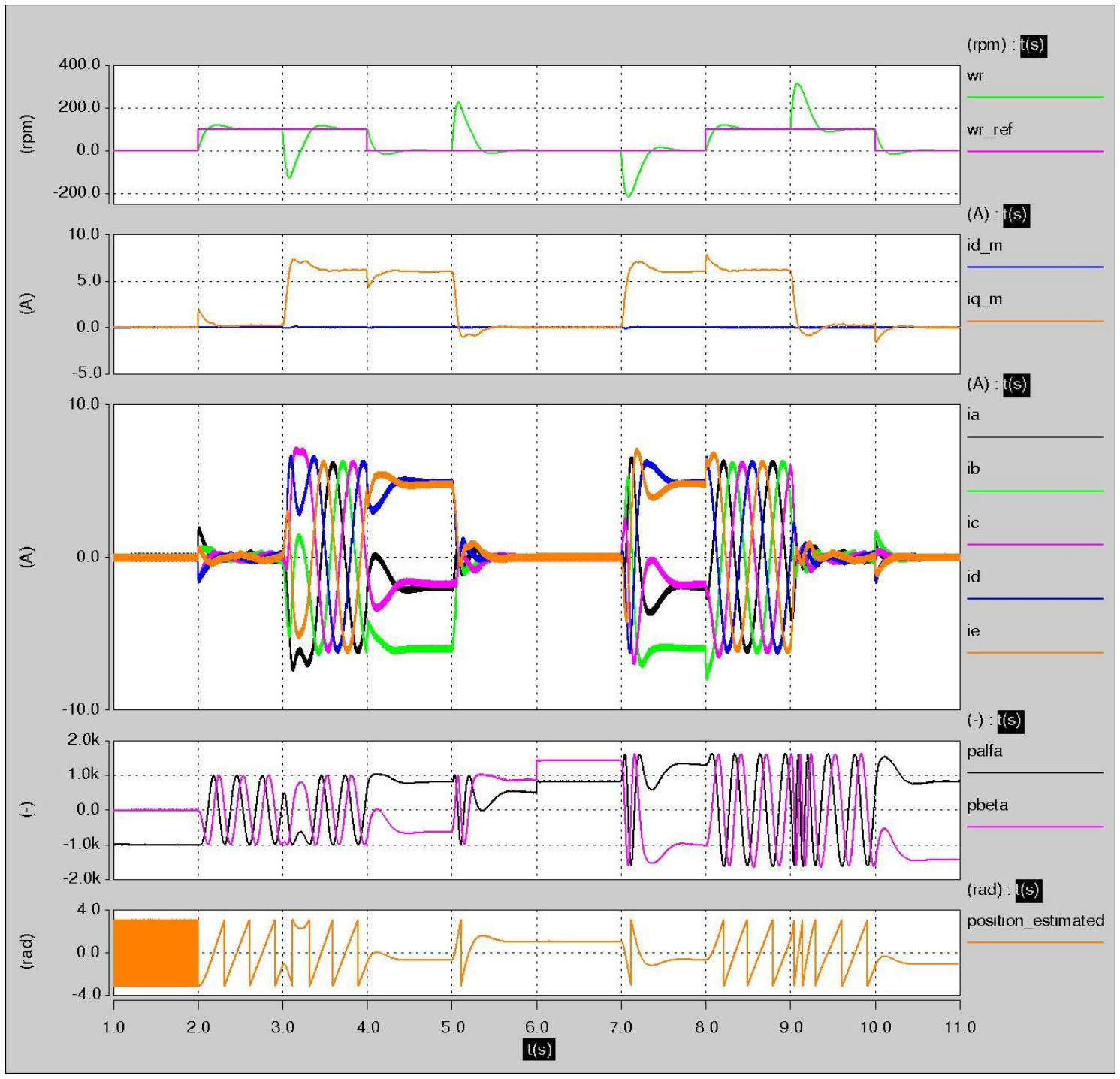

Figure 12 Fully Sensorless full load Steps.

\subsection{Current Distortion reduction}

The main difficulty when applying current derivative-based position estimation schemes to real systems arises from the parasitic effects within the motor, the inverter and the cabling [24]. And hence, narrow active voltage vectors must be extended to a minimum vector duration tmin which is set according to the high frequency decay. In this paper tmin is set to 10 us which means that the active vector V1 and V4 in case of using the algorithm given in table 1 (fig 13.a) and the active vectors V2 and V3 in case of the algorithm given in table 2 (fig 13.b) should be extended to 10 us if the time of duration of any of them is less than that as shown in fig 13.a and fig13.b. 

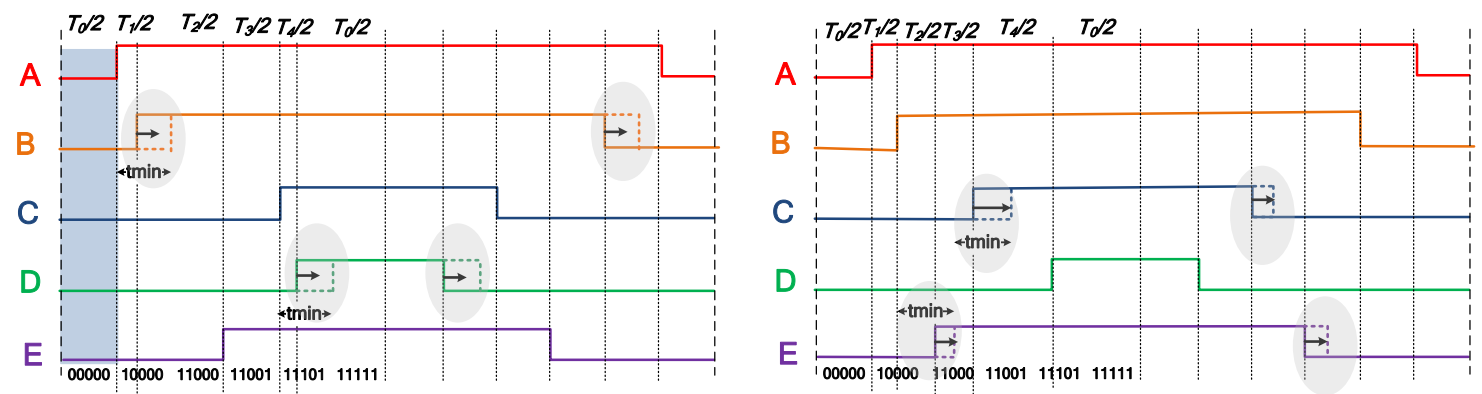

Fig 13 Space vector modulation state diagram for five phase inverter in case that $\mathrm{V}$ ref $\alpha 1-\beta 1$ exists in first sector a) $\mathrm{V} 1$ and V4 are extended as in table 1, b) V2 and V3 are extended as in table 2.

The time duration (T0,T1,T2,T3 and T4) of the active vectors shown in fig 13.a and 13.b are obtained using equations (7-9). It should be highlighted that when the phase voltage is sinusoidal, or the magnitude of the d3q3- axes voltage vector is zero as in our case, the following equations hold true [12]:-

$$
\begin{aligned}
T 3 & =1.618 * T 1 \ldots .(42) \\
T 2 & =1.618 * T 4 \ldots .(43)
\end{aligned}
$$

This means that the extension of the vectors V2 and V3 will be less than that of the vectors V1 and V4 which helps to reduce the current distortion introduced to the motor stator currents as shown in fig 14.a fig 14.b and fig 14.c. Fig 14.a demonstrate the sequence of applying the active vectors V1,V2,V3,V4 to generate the reference voltage Vref $\alpha 1-\beta 1$ if it exists in the first sector in the normal case (without extensions). In Fig $14 \mathrm{~b}$ the active vectors V1 and V4 are extended to tmin and finally in fig 14.c the active $\mathrm{V} 2$ and V3 are extended to tmin. From the figures it is quite clear that the voltage ripples (grey areas) in the case of extending the active vectors $\mathrm{V} 2$ and $\mathrm{V} 3$ is less than those in the case of extending the active vectors $\mathrm{V} 1$ and V4 and so less current distortion will be introduced to the motor stator currents.

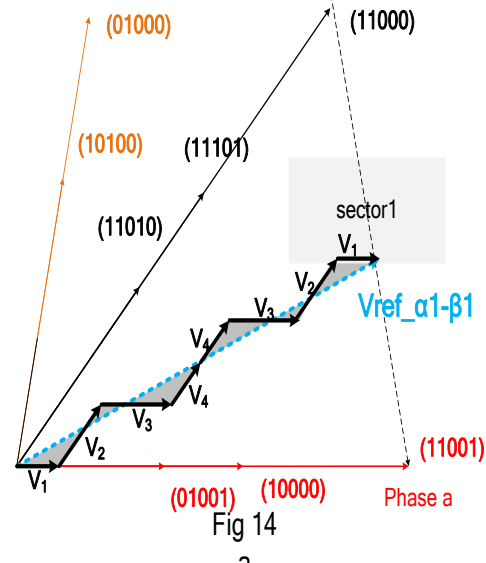

Fig 14. Current ripple for different extension schemes. a) no extensions. b) extension of active vectors V1 and V4. c) extension of active vectors V2 and V3.

The above idea is demonstrated using Saber simulator as shown in Fig 15. The motor was running at 400 rpm without any extensions to any of the active vectors. From $t=1.8 \mathrm{~s}$ to $2.1 \mathrm{~s}$ active vectors V1 and V4 are extended according to algorithm given in table 1. Finally from $t=2.1 \mathrm{~s}$ to the end active vectors V2 and V3 are extended according to algorithm given in table 2. It is clear from the waveform of the stator current ib that the extension of the active vectors V2 and V3 between $\mathrm{t}=2.1 \mathrm{~s}$ till $2.4 \mathrm{~s}$ is introducing less distortion to the stator current compared to the extension of the active vectors V1 and V4 between $\mathrm{t}=1.8 \mathrm{~s}$ and $\mathrm{t}=2.1 \mathrm{~s}$. This due to fact that between $\mathrm{t}=2.1 \mathrm{~s}$ and $\mathrm{t}=2.4 \mathrm{~s}, 10 \%$ of the PWM periods have at least active vectors (V2 or V3) longer than tmin while all PWM periods between $t=1.8$ to $t=2$.s need to extend both vectors V1 and V4. Moreover the length of the vector V2 and V3 as shown in Fig 15 are bigger than the vectors length of the active vectors $\mathrm{V} 1$ and $\mathrm{V} 4$ make the extension of the vectors $\mathrm{V} 2$ and $\mathrm{V} 3$ is less. 

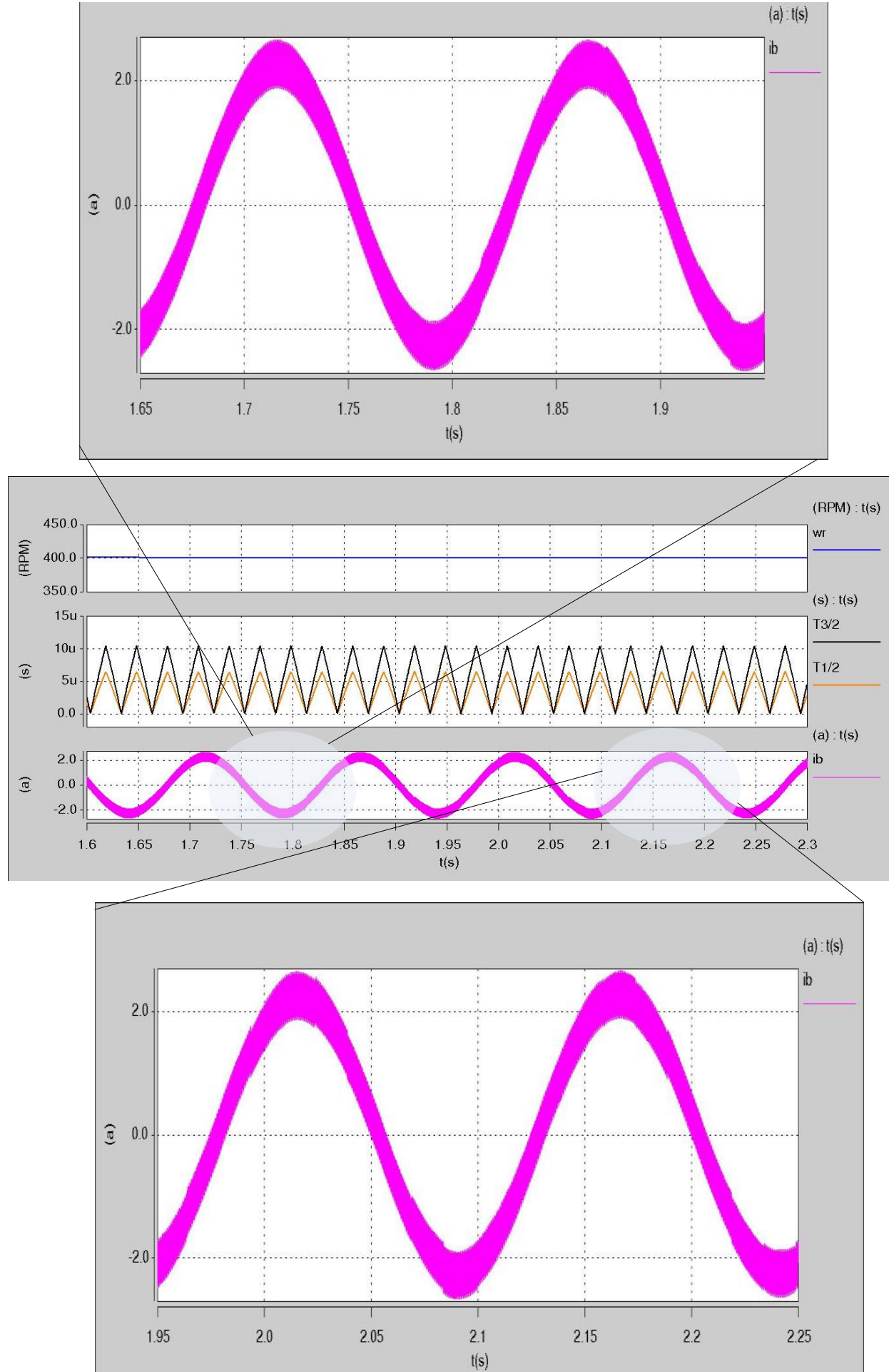

Fig 15 current waveform under different extension schemes

The FFT of the current wave forms in fig 15 is given Fig 16.a and 16.b and 16.c. The results proves the advantage of extension of V2 and V3 over extension of active vectors V1 and V4 in terms of current distortion. 

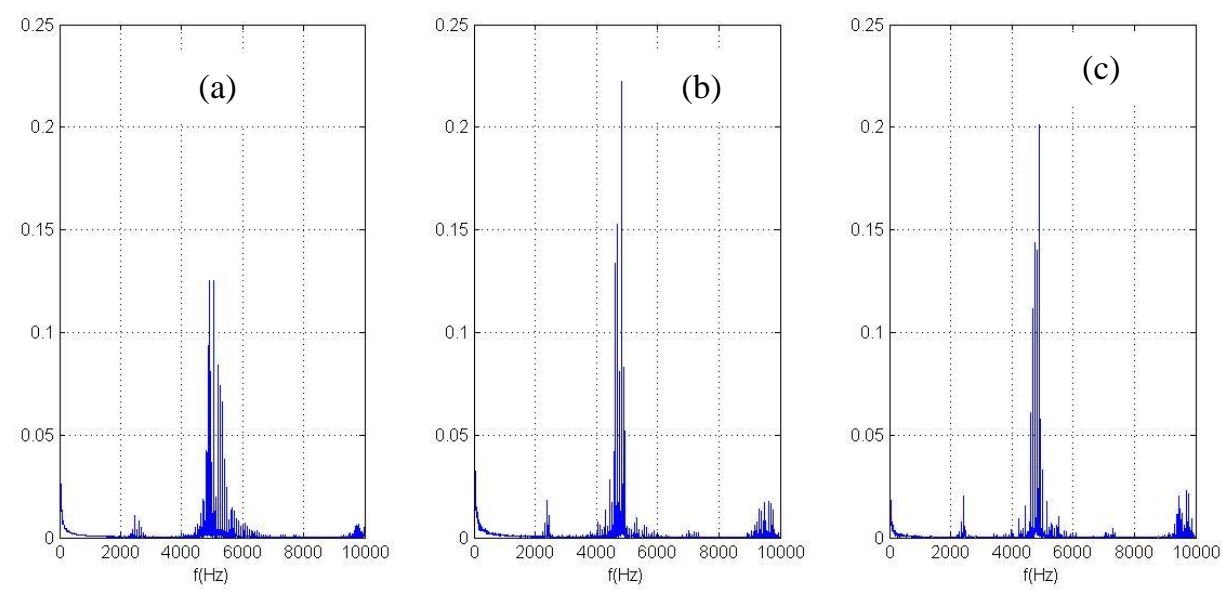

Fig 16 FFT of the current waveform under different extension schemes. a) no extensions b) extension of active vectors V1 and V4 c) extension of vectors V2 and V3.

\section{Conclusion}

This paper has outlined a two new algorithms for tracking the saliency of motor fed by a five-leg inverter in through measuring the dynamic current response of the motor line currents due the IGBT switching actions. The proposed methods include software modification to the method proposed in[18] to track the saliency of the motor fed by three phase inverter. The two new algorithms can be used to track the saturation saliency in PM and the rotor slotting saliency in induction motors. The results have shown the advantages of using the switching action of the active vectors V2 and V3 over using the active vectors V1 and V4 in terms of quality of the estimated position signals and the distortion introduced to the stator currents by extending the narrow vectors.

\section{References}

[1] Villani M, Tursini M, Fabri G, Castellini L. Multi-phase fault tolerant drives for aircraft applications. In: IEEE 2010 Electrical Systems for Aircraft, Railway and Ship Propulsion Conference (ESARS); 19-21 October 2010; Bologna, Italy. New York, NY, USA: IEEE. pp. 1-6.

[2] Qingguo S, Xiaofeng Z, Fei Y, Chengsheng Z. Research on space vector PWM of five-phase threelevel inverter. In: IEEE 2005 Electrical Machines and Systems conference (ICEMS); 29-29 September 2005; Nanjing, China. New York, NY, USA: IEEE. pp. 1418-1421.

[3] Ruhe S, Toliyat HA. Vector control of five-phase synchronous reluctance motor with space vector pulse width modulation (SVPWM) for minimum switching losses. In: IEEE 2002 Applied Power Electronics Conference and Exposition (APEC);10-14 March 2002; Dallas, Texas, USA. New York, NY, USA: IEEE. pp. 57-63.

[4]Abbas MA, Christen R, Jahns T.M. Six-phase voltage source inverter driven Induction motor. IEEE T Ind Appl 1984; 20: 1251-1259.

[5] Zhao X, Lipo TA. Space vector PWM control of dual three-phase induction machine using vector space decomposition. IEEE T Ind Appl 1995; 31:1177-1184.

[6] Parsa L, Toliyat HA. Multi-phase permanent magnet motor drives. In: IEEE 2003 Industry Applications Conference (IAS); 12-16 October 2003; Utah, USA. New York, NY, USA: IEEE. pp. 401408.

[7] Xu H, Toliyat HA, Pertersen LJ. Five-phase induction motor drives with DSP-based control system. IEEE T Ind Appl 2002; 17: 524-533.

[8] Kelly JW, Strangas EG, Miller JM. Multiphase space vector pulse width modulation. IEEE T Energy Conver 2003; 18: 259-264.

[9] Xue S, Wen X, Feng Z. A novel multi-dimensional SVPWM strategy of multiphase motor drives. In: EPE 2006 Power Electronics and Motion Control Conference; 30 August- 1 September 2006; Portoroz, Slovenia. Brigitte Sneyers, Pleinlaan 2, Brussels, Belgium: EPE. pp.931-935.

[10] Pengfei W, Ping Z, Fan W, Jiawei Z, Tiecai L. Research on dual-plane vector control of five phase fault-tolerant permanent magnet machine. In: IEEE 2014 Transportation Electrification Asia-Pacific conference (ITEC Asia-Pacific); 31 August- 3 September 2014; Beijing, China. New York, NY, USA: IEEE. pp. 1-5. 
[11] Minghao T, Wei H, Ming C. A novel space vector modulation strategy for a five-phase flux-switching permanent magnet motor drive system. In: IEEE 2014 Electrical Machines and Systems conference (ICEMS); 22-25 October 2014; Hangzhou, China. New York, NY, USA: IEEE. pp.1622-1628.

[12] Keng-Yuan Chen. Multiphase pulse-width modulation considering reference order for sinusoidal wave production. In: IEEE 2015 Industrial Electronics and Applications conference (ICIEA); 15-17 June 2015; New Zealand, Auckland. New York, NY, USA: IEEE. pp.1155-1160.

[13 ] Z.M.S.El-Barbary. DSP Based Vector Control of Five-Phase Induction Motor Using Fuzzy Logic Control. International Journal of Power Electronics and Drive System (IJPEDS) Vol.2, No.2, June 2012, pp. 192 202

[14] Atif Iqbal, Shaikh Moinoddin*, Khaliqur Rahman. Finite State Predictive Current and Common Mode Voltage Control of a Seven-phase Voltage Source Inverter. International Journal of Power Electronics and Drive System (IJPEDS) Vol. 6, No. 3, September 2015, pp. 459 476

[15] Olivieri C, Fabri G, Tursini M. Sensorless control of five-phase brushless DC motors. In: IEEE 2010 Sensorless Control for Electrical Drives conference (SLED); 9-10 July 2010; Padova, Italy. NewYork, NY, USA: IEEE. pp.42-31.

[16] Karampuri R, Prieto J, Barrero F, Jain S. Extension of the DTC technique to multiphase induction motor drives using any odd number of phases. In: IEEE 2014 Vehicle Power and Propulsion Conference (VPPC); 27-30 October 2014; Coimbra, Portugal. NewYork, NY, USA: IEEE. pp.1-6.

[17] Parsa L, Toliyat HA. Sensorless direct torque control of five-phase interior permanent-magnet motor drives. IEEE T Ind Appl 2007; 43: 952 - 959.

[18] wubin K, Jin H, Ming K, Bingnan L. Research of sensorless control for multiphase induction motor based on high frequency injection signal technique. In: IEEE 2011 Electrical Machines and Systems conference (ICEMS); 20-23 August 2011; Beijing, China. NewYork, NY, USA: IEEE. pp.1-5.

[19] Minglei G, Ogasawara S, Takemoto M. An inductance estimation method for sensorless IPMSM drives based on multiphase SVPWM. In: IEEE 2013 Future Energy Electronics Conference (IFEEC); 3-6 November 2013; Tainan, Taiwan. NewYork, NY, USA: IEEE. pp.646-651.

[20] Schroedl M. Sensorless control of AC machines at low speed and standstill based on the INFORM method. In: IEEE 1996 Industry Applications Conference; 6 -10 October 1996; San Diego, USA. New York, NY, USA: IEEE. pp. 270-277.

[21] Holtz J, Juliet J. Sensorless acquisition of the rotor position angle of induction motors with arbitrary stator winding. In:IEEE 2004 Industry Applications Conference; 3-7 October 2004; Washington, USA. NewYork, NY, USA: IEEE. pp.1321-1328.

[22] Qiang G, Asher GM, Sumner M, Makys P. Position estimation of AC machines at all frequencies using only space vector PWM based excitation. In: IET 2006 International Conference on Power Electronics, Machines and Drives; 4-6 April 2006; Dublin, Ireland. Savoy Place, London, UK: IET.pp. 6170 .

[23] Lorenz RD, Van Patten KW. High-resolution velocity estimation for all-digital, ac servo drives. IEEE T Ind Appl 1991;27:701-705

[24] Y. Hua, M. Sumner, G. Asher, Q. Gao and K. Saleh, "Improved sensorless control of a permanent magnet machine using fundamental pulse width modulation excitation," in IET Electric Power Applications, vol. 5, no. 4, pp. 359-370, April 2011. 